While one practitioner regards arsenic as a specific for true chorea, another cures nearly all his cases with the sulphate or the oxide of zinc, and another considers the sulphate or the carbonate of iron as an almost infallible remedy. This may perhaps arise, in a great measure, from habit. It is possible that all may be nearly equally efficacious. I have seen each of these, and many other remedies cure the complaint; and 1 have seen them all occasionally fail.",

Zinc has been given in large doses, especially at Guy's Hospital; where Dr. Hughes has stated that thirty-six grains of the sulphate have been administered daily. It appears such an abuse of medicine, to prescribe these enormous doses of an irritant drug commonly used in such quantities as an emetic, that we are at loss to discover how it should ever have been thus given. What Sir John Forbes has termed the autocracy of Nature, could not have been understood by those prescribing it in such a manner; and Dr. Hughes observes :

"In the practice of the hospital, I am not aware that it has been shown that small or moderate doses of the mineral, continued an equally long time, will not effect a cure."

In my own practice, I have used iron and arsenic more frequently than zinc ; and I consider that, of the metallic tonics, iron is the best, and that arsenic stands next to it.

Cod-liver oil is a remedy which is sometimes of service, by improving the general health; and it may be given with, or subsequent to, the preparations of iron. Electricity seems, in some cases, to have acted beneficially, and in a very rapid manner.

The shower-bath is a remedial agent which gives tone to the system, and may be used along with other tonic treatment. I have seen it of the greatest use in many cases. In the case of a young lady, in whom the movements were incessant and violent, and prevented sleep, the movements subsided very much directly after the bath, and she soon fell asleep, and ultimately recovered. The emaciation and exhaustion of the patient from the incessant violent movements and the loss of sleep, had rendered the recovery of this patient very doubtful.

It is not less important to glance now at the means which may prove detrimental, and which should therefore be avoided in the treatment of this nervous affection. All violent impressions on the nervous system should be guarded against. We know that fright is not an unfrequent cause of the disease. It is probably on this account that the shower-bath may prove injurious when injudiciously employed, as well as very frequently useful. Narcotic remedies, such as opium, morphia, and hyoscyamus, have appeared to me (contrary to what might be expected) to be not simply useless, but positively hurtful. It has been said that chloroform is of use but I saw the inhalation tried in two very severe cases, which proved fatal; and though it produced a temporary sedative influence, it appeared to me to act injuriously on the general progress of the disease.

In all cases, due attention should be paid to hygienic as well as medicinal treatment, so as to improve in every way practicable the nutritive functions and the general health. The diet should be nutritious, and wine or malt liquors are often of service; and out-door exercise is desirable in the less severe cases. When the disease proves chronic, change of air is advisable; and in the summer the change may be made with advantage to the sea-side, where seabathing may be practised.

\section{ON THE PHYSICS OF DISEASE, AND THE PHYSICAL PATHOLOGY OF THE BLOOD.}

By Benjamin W. Richardson, M.A., M.D., Senior Physician to the Royal Infirmary for Diseases of the Chest.

\section{Chapter II.}

The Force evolved in Oxygenation of Blood. Conditions of Oxygen and of Blood that modify Combination. Modifications of Oxygen-Ozone. Influence of Temperature.

I REFERRED in my last chapter to the two modes, external and internal, by which force or motion is communicated to the body. When we view the subject free from all prejudice and all superstition, we are driven to the conclusion that by none other than the means there described can motion be supplied: To presume that the nervous system can generate motion, that it can out of nothing produce something, is like saying that a galvanic battery may work a telegraph without chemical action. The nervous system is the centre of forces derived from without and within-the tabula rasa on which the external world is written - the centre in which motion derived from the blood is laid up, to be applied in volition. But, beyond this, the nervous system is not different from other parts of the physical organism.*

Let us then consider the source of that motion which springs from within the body; which, in better words, is evolved in the body; which gives animal power, but does not give intelligence; which makes the animal an engine, but not an animal; which is constructive and locomotive, but not animating ; but which yet prepares the machine for animation, and without which there is no life.

. This force or motion is elicited by the combination of the oxygen of the air with the carbon derived from the vegetable kingdom, and present in solution or suspension in the blood. We speak of the force thus evolved as primary in respect to the body, and so it is; but, in truth, we may trace it back further-at least, to the sun.

When the sunbeam falls on the vegetable world, when the radiant motion from the sun touches the vegetable world, that chemical change takes place in the leaf of the plant which is described as consisting of the decomposition of carbonic acid, the evolution of oxygen, and the fixation of carbon. This is the chemical part of the change that takes place; but there is a physical part played as well. The motion derived from the sun is also fixed, to be laid by until it is required, until the time has come when, by the recombination of oxygen with carbon, it shall be set at liberty in the form of heat, as heat of fire, or heat of body. Thus the force that supplies the organism with the motion that pertains strictly to its organic or vegetative life is conveyed into the body as coal is conveyed to the fire, through the vegetable worldconveyed as fuel to the furnace, and as food to the animal. Plants are condensers of motion for man; they take up from the earth the dead matter; they take from the sun motion; they endow the dead matter with motion; and, thus endowing it, they become to the animal constructively and actively as matter and motion.

When distributed through the blood, the carbon from the plant meets, in infinite subdivision of mole-

* In a paper read before the Medical Society of Iondon in the beginning of 1863 , I described the nervous system as the condenser of motion. 
cule, the oxygen of the air, that being also in infinite subdivision: then those processes which took place when the sun's motion touched the plant, are reversed. Carbon and oxygen reunite; carbonic acid is reformed for the plant; and motion-heat-is evolved. Nor is the motion merely evolved to the body, but through the body it is given forth again to the universe in act-nay, in thought. This that $I$ write is so much motion fixed through matter on the page; and when the reader, in presence of light, takes it up and reads it, it reaches him as motion liberated and carried to the eye by light, another form of motion.

To maintain a perfect balance of motion, either in the vegetable or animal world, there are conditions necessary, a departure from which is at once followed by organic change. If we deprive a vegetable of the chemical radiations of the sunbeam, we destroy, to the extent of the deprivation, the absorption of motion; or if we deprive the plant of its green colouring matter, we destroy, to the extent of the deprivation, the reception of motion. On the other hand, if we deprive an animal of oxygen, we destroy, to the extent of the deprivation, the liberation of motion; and, if we deprive it of food, we do the same thing.

This is simple enough; and, in the case of the animal, we see the phenomenon almost daily of death or cessation of motion from mere deprivation of oxy. gen, or from mere deprivation of food or blood. We see the first in what is called asphyxia; the second, in what is called hæmorrhage.

But between perfect combination, or health, and stopped combination, or death, there are numerous gradations, depending either on modifications in the conditions of oxygen alone, of blood alone, or of both oxygen and blood. In the presence of these modified conditions, the animal combustion is not suspended, but lessened or increased, with, in some instances, the production of new products of combustion which are foreign to the organism. These modified conditions are the causes of those various groups of symptoms which we call diseases; and on them all diseases rest, except a few, of which I shall speak in a future chapter, and which are mechanical in their origin, such as intussusception, hydatid disease, and some kinds of external injury.

MODIFIED CONDITIONS OF OXYGEN.

Let us turn first to oxygen, and ascertain what are the modified conditions under which it fails to sustain in proper degree the animal fire.

In order that oxygen should combine with blood, it is an essential condition that its molecules should be distributed, held apart from each other, and possess in themselves motion; hence a given external temperature is necessary for the oxidation of blood. In the next place, it is essential that the oxygen should be diluted in nitrogen; for, if this did not obtain, variations of temperature would lead to sudden and immediate consequences of the most serious kind. A fall in temperature below $35^{\circ} \mathrm{Fahr}$. would stop combustion altogether; an elevation in temperature to $110^{\circ} \mathrm{Fahr}$. would give rise to fatal combustion from excess of action. In the third place, the oxygen must be presented to the lung in the neutral form. Raised in action by being, in very minute proportions, transformed into ozone, it is unfitted for respiration : it becomes an irritant to the mucous membranes over which it passes to the blood, a quickener of action, and, by this quickened action, a disorganiser of the blood. Reduced, on the opposite side, to a negative condition, it fails in combining power, so that the blood exposed to it is unable to supply sufficient motion to the organism to sustain the functional activity.

In order to determine the effects of modified condi- tions of oxygen on the combustion of blood, I have conducted two series of inquiries. In one series, animals have been subjected, while they were yet in motion-i.e., living-to oxygen in various states; in another series, blood that has been removed from an animal has been subjected, spread over a very wide surface, to oxygen in various states. It will be best to first direct attention to the results of experiments on the living process.

INFLUENCE OF HEAT ON OXYGEN.

By motion in the form of heat, oxygen gas is held in varying conditions for combination; the rule being that the expansion of the gas by heat, within ordinary natural temperatures, quickens the process of oxidation in animal bodies.

In order to see the influence of varying degrees of heat on oxygen, in relation to its power as a supporter of life, it is best to place animals in an atmosphere of pure neutral oxygen, and then, the gas used being always talien from the same source, to cause its inhalation under varying degrees of temperature. By this method a series of results are obtained, on similar classes of animals, so uniform, that we may consider we have in our possession the main facts bearing upon the subject in a physiological point of view. My own inquiries have been conducted on rabbits and mice. In subjecting the rabbits to experiment the oxygen was breathed by the trachea; and in order to produce various temperatures of the gas inhaled, the tube leading from the jar of oxygen to the animal passed through thirty-six feet of thin metallic tubing, which, being coiled, could be immersed in a bath containing water at various temperatures, or ice and salt: by these means I could reduce the oxygen to $20^{\circ} \mathrm{Fahr}$., or raise it to $140^{\circ}$. In subjecting the mice to experiment, they, being small animals, were placed in chambers of known capacity, charged with oxygen and very carefully closed : by a simple arrangement the animals could be introduced into these chambers without water and without the admission of air. The chambers being thus prepared, the temperature of the air within them was modified by placing them in water heated or cooled to the degree required, ice and salt being used for the lowest temperatures. A thermometer in the chamber with the animal told the temperature of the enclosed air; and a very simple valve regulated the pressure of the gas, allowing escape when there was expansion, and means of entrance for oxygen when the extreme of cold was being applied.

When experiments constructed on the basis given are performed with exactness and without hurry, the uniformity of result is remarkable. I began by studyinc the effects of a low temperature, reducing the thermometer to $20^{\circ}-25^{\circ} \mathrm{Fahr}$. by means of ice and salt. I found that oxygen thus reduced was destructive both to the rabbits and the mice, in periods varying from fifteen to thirty minutes. The mice in all cases lived the longest, a result due probably to the mode of administration. The form of death is always the same; the animals became drowsy, and then slowly and regularly the action of the heart declines: there is no excitement of the heart first-no fluttering; it is a gradual arrest of motion; each minute brings two or three beats less, and so down and down, until the organ is quite at rest.

The animals die in these experiments because, under the influence of the cold, the combining power of the oxygen with the blood is reduced. I thought at one time it might be that the cold, by producing contraction of the capillary vessels of the lung, was the cause of death; and I, therefore, varied the experiment by subjecting animals to oxygen diluted as in air, but at the same reduced temperature. The 
result was that, in the diluted oxygen, a rabbit breathing by the trachea would live one-third longer than another one would by breathing oxygen alone. Two rats placed in similar chambers, and exposed to the same temperature, were also tested; one being subjected to pure neutral oxygen, the other to common air. The rat in oxygen had ceased to breathe in half an hour; the rat in air continued to breathe slowly and stertorously for nearly two hours: taken out at the end of that time, it was as insensible to all pain as though it were profoundly narcotised with chloroform, the cold producing general anæsthesia, as by Dr. James Arnott's beautiful process it is made to produce local anæsthesia. A three-inch spark from the large induction coil, passed through the limbs, produced no contraction; and yet, by very gradual exposure to warmth, the animal recovered.

It is not, therefore, from the mere constringing influence of cold exerted on the capillaries, that animals in pure oxygen die at low temperatures, although this, as a secondary cause, is important; it is that the abstraction of caloric from oxygen is more rapid and perfect when the gas is pure; diluted with nitrogen, as it is in air, the molecules of oxygen are not so readily brought within the sphere of attraction for each other, inasmuch as the nitrogen shares with the oxygen the abstraction of heat. In this sense, nitrogen in the air does more than simply dilute oxygen : it equalises it in action during extremes of heat and cold.

From temperatures ranging from $20^{\circ}$ to $25^{\circ} \mathrm{Fahr}$., I passed to those ranging from $50^{\circ}$ to $55^{\circ}$, subjecting animals, now in closed chambers only, to oxygen, neutral and pure. In these cases, in the same chambers, and breathing gas derived from the same source, the animals, instead of dying in thirty minutes at most, lived four hours-rather more than two hours longer than they would have lived had they been piaced in the same volume of common air at $55^{\circ} \mathrm{Fahr}$. ; for, although animals in oxygen, as in air, become comatose at the same periods, those in air die so soon as they have become comatose, while those in oxygen live for more than twice the same length of time.

From $50^{\circ}-55^{\circ}$ Fahr., we pass to $70^{\circ}-75^{\circ}$. Using the same chambers, using oxygen derived from the same source, and using animals of the same kind, I placed the animals in the chambers, and only modified the experiment by putting the chambers in such position that the thermometer told off a temperature within them of $70^{\circ}$, rising occasionally to $75^{\circ}$, but soon reduced. In this case, the animals remained conscious for three hours; then they became comatose ; but they continued to live for so long a period as twelve hours.

At temperatures of $80^{\circ}-90^{\circ}$, and even $100^{\circ}$, the effect produced on oxygen is not remarkably different from that observed in the last named experiments; but beyond $100^{\circ}$ the difference is very great. If the chamber containing the animal exposed to pure oxygen be placed so that the contained gas be gently raised to $125^{\circ} \mathrm{Fahr}$. (expansion of course being provided for), the animal lives for a period of fifteen minutes, breathing quickly, and becoming in the transparent parts of its body intensely red in colour ; at last, the redness quickly subsides into a purple tint, after which the animal falls unconscious, becomes tetanically convulsed, and in the course of three or four minutes dies.

It might occur to the reader, that the effects produced in the animals placed in confined chambers were due to the accumulation of carbonic acid. To remove this impression, it is necessary to direct attention to the air remaining in the chambers. In the chambers containing the oxygen inhaled by the animals at $20^{\circ}-25^{\circ} \mathrm{Fahr}$., the consumption of the gas by combustion is so small that only the mere presence of carbonic acid can be detected. To test the supporting power of this oxygen, I placed in a chamber containing it, after the first animal was dead, a second animal. I then gradually transferred the chamber from a temperature of $25^{\circ}$ to a temperature of $70^{\circ}$. In another chamber of the same size, and filled with fresh oxygen, I placed a second animal, also at $70^{\circ}$, the chambers standing side by side. The two animals became comatose at the same time, and died within six minutes of each other; the one that had been placed in the chamber containing the dead animal dying first.

In a chamber in which an animal has been exposed to oxygen at $55^{\circ}$, the amount of gas consumed is also remarkably small. In a comparative experiment, in which two animals were subjected, one to pure oxygen, the other to common air, each chamber being of the same size, and the temperature being regulated in both at $55^{\circ}$, I found the animal that died in air had produced, although it breathed for two hours less, tive times the amount of carbonic acid. But in a chamber in which an animal had breathed the gas for twelve hours at $70^{\circ}-75^{\circ}$, the amount of carbonic acid, after the death of the animal, was found equal to that which was obtained from the respiration of the same quantity of common air by another similar animal. In fact, the animal in this case dies, although after a period six times prolonged, in the same manner as after the inhalation of the same volume of common air-viz., from the accumulation of carbonic acid.

In chambers in which animals die from breathing oxygen at $125^{\circ}$, the amount of carbonic acid is variable: it is always greater than would be developed from air respired during the same period, but all the oxygen is not consumed; and the jar, after the animal is dead, contains as much as will sustain, at a temperature of $70^{\circ}$, another animal for two hours.

Thus we see that extremes of heat and of cold render oxygen incapable of supporting life. The low temperature prevents, the high temperature overquickens, oxidation; while, at mean temperatures, the pure gas sustains for a long time a slow process of oxidation, during which the organic, as apart from the conscious life, is maintained; during which just sufficient motion is generated from combustion to support the heart and respiration, but none is made to be stored up in the nervous centres.

In my next chapter, I shall describe at some length certain new experimental facts relating to active oxygen-ozone-in regard, specially, to its effects on the organism.

Bequest. Edward Yates, Esq., leaves by will so much of his property as may legally be applied to charitable purposes to University College, London, in trust, to apply one moiety of the annual income thereof to the general purposes of the North London Hospital, connected with that college, and the other moiety to the Samaritan fund for the relief of poor patients. The residue of personal property is left to Dr. C. J. Hare, one of the executors.

Death of Dr. Barkie. Sir R. Murchison announces that Dr. Baikie died at Sierra Leone, on November 30 th, of fever and dysentery. The death of this good and distinguished man will be as deeply deplored by ceographers and naturalists who have been daily looking out for his arrival in England, charged as he was with much important knowledge and rich collecctions relating to the interior countries on the banks of the Niger, as he will be by the government which he served during nearly eleven years with so much ability and zeal in carrying out his arduous mission. 